\title{
Role of miR-128 in hypertension-induced myocardial injury
}

\author{
JIE YIN ${ }^{1}$, HONGYAN LIU ${ }^{1}$, LEI HUAN ${ }^{1}$, SUPING SONG ${ }^{2}$, LIYING HAN ${ }^{3}$, FAXIN REN $^{4}$, \\ ZENGTANG ZHANG ${ }^{1}$, ZHIQIANG ZANG ${ }^{1}$, JUNYE ZHANG ${ }^{5}$ and SHU WANG $^{6}$ \\ ${ }^{1}$ Department of Cardiology, Laiwu People's Hospital; ${ }^{2}$ Department of The Second Medicine, \\ Laiwu People's Hospital, Laiwu, Shangdong 271100; ${ }^{3}$ Ordance Industrial 521 Hospital, Xi'an, \\ Shanxi 710000; ${ }^{4}$ Department of Cardiology, Yuhangding Hospital of Yantai, Yantai, Shangdong 264000; \\ ${ }^{5}$ Laboratory of Cardiac Function, Qilu Hospital of Shangdong University, Jinan, Shandong 250012; \\ ${ }^{6}$ Sino German Laboratory, Fuwai Hospital, Beijing 100037, P.R. China
}

Received July 24, 2015; Accepted January 13, 2017

DOI: 10.3892/etm.2017.4886

\begin{abstract}
The present study aimed to investigate the role and mechanism of micro RNA (miR)-128 in hypertension-induced myocardial injury. The peripheral blood of patients with hypertension was collected and the expression of miR-128 was detected using fluorescence reverse transcription-quantitative polymerase chain reaction (RT-qPCR). Primary myocardial cells isolated from rat in vitro were cultured under conditions of hypoxia and glucose deprivation, and miR-128 expression was measured by RT-qPCR. The expression of c-Met protein was measured using western blot analysis and the apoptosis of transfected cells was measured by flow cytometry in rat myocardial cells following transfection with miR-128 mimics or c-Met siRNA. A luciferase assay was applied to assess the binding of miR-128 to c-Met mRNA. miR-128 expression was significantly higher in hypertension patients compared with controls $(\mathrm{P}<0.05)$. miR-128 expression was higher in patients with stage III/IV hypertension compared with patients with stage II hypertension. Similarly, miR-128 expression in primary cardiomyocytes cultured under deprivation of oxygen and glucose increased with the culture time and reached a peak at $12 \mathrm{~h}$. c-Met expression decreased significantly $(\mathrm{P}<0.05)$ and the ratio of apoptotic cells increased significantly $(\mathrm{P}<0.05)$, following transfection of miR-128 mimics. The number of apoptotic cells also increased when c-Met expression was knocked down by siRNA. The dual luciferase assay indicated that fluorescence intensity decreased significantly in miR-128 mimics and wild type c-Met group $(\mathrm{P}<0.05)$, indicating that miR-128 can directly target c-Met. Therefore, the results of the current study suggest that miR-128 may promote myocardial cell injury by regulating c-Met expression.
\end{abstract}

Correspondence to: Professor Jie Yin, Department of Cardiology, Laiwu People's Hospital, 1 Snow Lake Avenue, Laiwu, Shangdong 271100, P.R. China

E-mail: cqx333@126.com

Key words: miR-128, myocardial injury, c-Met, hypertension

\section{Introduction}

Hypertension is one of the commonly diagnosed cardiovascular diseases in the clinic and its incidence rate increases every year $(1,2)$. It can induce severe myocardial injury, heart failure, stroke and other complications, which pose great threats to human health $(3,4)$. Sustained increases in blood pressure can damage myocardial cells and cause abnormal cardiac reconstruction (5). Myocardial injury induced by hypertension is characterized by cardiac hypertrophy and cardiac fibrosis, which are the main causes of chronic heart failure $(6,7)$. Hypertension can induce myocardial injury through a variety of mechanisms, including neural, humoral and immune inflammatory reactions, and myocardial injury has great value in the diagnosis and treatment of hypertension $(8,9)$. In recent years, it has been demonstrated that micro RNA (miRNA) serves important roles in the process of abnormal cardiac reconstruction induced by hypertension $(10,11)$. However, the mechanism of how miRNA regulates this process remain unclear.

miRNA is a type of small non-coding RNA 22 nucleotides long, which serves an important post-transcriptional role. It has been demonstrated that miRNA regulates the growth, differentiation and injury of cardiac cells, and thus has important clinical value $(12,13)$. Witman et al $(14)$ determined that miR-128 serves an important role in myocardial cell hyperplasia by regulating the expression of Islet1 in newts. However, it remains unknown how miR-128 acts in myocardial injury induced by hypertension. Bioinformatics predictions have suggested that c-Met is a candidate target gene of miR-128, indicating that miR-128 may regulate the hepatocyte growth factor (HGF)-c-Met signaling pathway (15). It has been observed that HGF can promote the proliferation of endothelial cells in the myocardial infarction ischemic area and accelerate the formation of new capillaries (16). The c-Met protein is a high affinity receptor of HGF, which can transfer the cell signals after binding with HGF (17).

The current study aimed to investigate the role and the mechanism of miR-128 in hypertension induced myocardial injury. The results of the current study may provide experimental evidence and theoretical guidance for understanding 
the molecular mechanisms of hypertension in the induction of myocardial injury.

\section{Materials and methods}

Patients. The current study included 52 patients with primary hypertension admitted to the department of cardiology at Laiwu City People's Hospital (Laiwu, China) between December 2012 and January 2014. Among them, there were 33 males and 19 females aged 63-71 years old (mean age, 68.9 years old; median age, 67 years). Their peripheral blood was collected. All patients had suffered from primary hypertension $>5$ years (hypertension was defined as systolic pressure $140-159 \mathrm{mmHg}$, diastolic pressure 90-99 $\mathrm{mmHg}$ ). Patients with other chronic diseases were excluded from the present study. According to the clinical grading standard of hypertension (18), there were 28 patients with grade II hypertension, 14 patients with grade III hypertension and 10 patients with grade IV hypertension. For controls, 20 healthy volunteers (mean age, 35 years) were recruited during the same time period and their peripheral blood was collected. Prior written and informed consent was obtained from every patient and the present study was approved by the ethics review board of Laiwu City People's Hospital.

Isolation and culture of primary myocardial cells. Primary myocardial cells were isolated from newborn Sprague-Dawley rats $<3$ days old (Chengdu Dossy Experimental Animals Co., Ltd., Chengdu, China). Rats were sacrificed by cervical dislocation and the apex portion of the heart was harvested by cutting open the left chest wall along the midline of the sternum. The heart was cut into $1 \mathrm{~mm}^{3}$ pieces using ophthalmic scissors and then washed twice with magnesium-free PBS. Following digestion with trypsin and collagenase, myocardial cells were collected and centrifuged at $200 \mathrm{x} g$ at room temperature for $5 \mathrm{~min}$. The collected cells $\left(1 \times 10^{5}\right.$ cells/well $)$ were cultured in high glucose Dulbecco's Modified Eagle's Medium (H-DMEM; BD Biosciences, Franklin Lakes, NJ, USA) with $10 \%$ fetal bovine serum (FBS; BD Biosciences) in an incubator at $37^{\circ} \mathrm{C}$ with $5 \%$ $\mathrm{CO}_{2}$ for $48 \mathrm{~h}$. To induce hypoxia, $1 \times 10^{5}$ cells/well were incubated in glucose-free DMEM with $1 \% \mathrm{O}_{2}$ and $5 \% \mathrm{CO}_{2}$ for $24 \mathrm{~h}$.

RNA extraction from peripheral blood. The peripheral blood of patients was centrifuged at 2,000 x g for $10 \mathrm{~min}$ at room temperature. A total of $250 \mu 1$ serum in supernatant was thoroughly mixed with $750 \mu 1$ TRIzol $^{\circledR}$ (Invitrogen; Thermo Fisher Scientific, Inc., Waltham, MA, USA) and RNA was extracted by phenol-chloroform methods as previously described (19). RNA quality was checked by gel electrophoresis and the absorbance ratio was measured at 260 and $280 \mathrm{~nm}$ using a spectrophotometer. miRNA cDNA was reversely transcribed using the PolyA tailing method according to the manufacturer's protocol and cDNA was stored at $-20^{\circ} \mathrm{C}$. The procedure for reverse transcription of miRNA followed the protocol provided with the miScript SYBR Green PCR kit (catalogue number: 218160; Qiagen GmbH, Hilden, Germany): $6 \mu$ RNA template, $2 X$ miRNA Reaction Buffer Mix $10 \mu l, 2 \mathrm{ml} 0.1 \%$ bovine serum albumin, $2 \mathrm{ml}$ miRNA PrimeScript RT Enzyme Mixture (Takara Biotechnology, Co., Ltd., Dalian, China). The reaction was performed at $37^{\circ} \mathrm{C}$ for 60 min with PolyA primer (included in the miScript kit) and the total reaction mixture volume was $20 \mathrm{ml}$.
To extract total RNA from primary cardiomyocytes, myocardial cells incubated under deprivation of oxygen and glucose were collected following $0,2,4,8,12$ and $24 \mathrm{~h}$ culture. As mentioned previously, TRIzol and phenol-chloroform methods were used to extract total RNA. The reverse transcription protocol was same as that followed for peripheral blood.

Fluorescence quantitative polymerase chain reaction ( $q P C R)$. The SYBR Green Master Mix for real-time PCR (Takara Biotechnology, Co., Ltd) was used to detect the expression of miR-128 in peripheral blood and primary cardiomyocytes, following the manufacturer's protocol. The reaction system included $10 \mu \mathrm{l}$ SYBR Green Master Mix, $0.5 \mu$ l forward primer

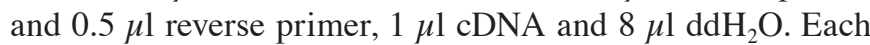
sample had 3 replicates. The cycle conditions were as follows: $95^{\circ} \mathrm{C}$ for $15 \mathrm{sec}$, followed by 40 cycles at $95^{\circ} \mathrm{C}$ for $15 \mathrm{sec}$ and $60^{\circ} \mathrm{C}$ for $30 \mathrm{sec}$. The results were calculated using the $2^{-\Delta \Delta \mathrm{Cq}}$ method and U6 was used as internal reference (20). The primer sequences were as follows: U6, forward 5' CTCGCTTCGGCA GCACA 3' and reverse 5'AACGCTTCACGAATTTGCGT3'; miR-128, forward 5'-UCACAGUGA ACCGGUCUCUUU-3' and the reverse primer was included in the miScript II RT kit without sequence information.

Transfection of miR-128 mimics and c-Met small interfering (si)RNA in myocardial cells. Primary cultured myocardial cells were divided into three groups: Cells transfected with miR-128, cells transfected with c-Met siRNA and negative control (NC, transfected with miR-NC) group. For transfection of miR-128 mimics, cells $\left(1 \times 10^{5}\right.$ cells/well $)$ were cultured in antibiotic-free H-DMEM medium with $10 \%$ FBS. miR-128 mimics (1.25 $\mu$ l; Hanbio Technology Co., Ltd., Shanghai, China) and $1 \mu \mathrm{l}$ Lipofectamine ${ }^{\circledR} 2000$ (Invitrogen; Thermo Fisher Scientific, Inc.) were added to eppendorf tubes containing $50 \mu 1$ Opti-MEM medium (Invitrogen; Thermo Fisher Scientific, Inc.), respectively. The two tubes were mixed together after $5 \mathrm{~min}$ incubation and then incubated for a further $20 \mathrm{~min}$ at room temperature. The mixture was added to each well. After $6 \mathrm{~h}$ transfection, medium was replaced with H-DMEM medium containing $10 \%$ FBS.

c-Met siRNA (Hanbio Technology Co., Ltd.) was transfected into primary myocardial cells based on same protocol. c-Met protein expression was subsequently measured following $48 \mathrm{~h}$ transfection with miR-128 mimics or c-Met siRNA.

Western blot analysis. Following transfection for $48 \mathrm{~h}$, myocardial cells were collected to detect the changes in c-Met expression by western blotting. Collected cells were washed twice with precooled PBS and then lysed at $4^{\circ} \mathrm{C}$ for $30 \mathrm{~min}$ with radioimmunopreciptation assay buffer (Beyotime Institute of Biotechnology, Beijing, China) containing $1 \%$ phenylmethane sulfonyl fluoride (Beyotime Institute of Biotechnology, Beijing, China) to extract the total proteins from these cells. The lysate was the centrifuged at $12,000 \mathrm{x} \mathrm{g}$ for $15 \mathrm{~min}$ at $4^{\circ} \mathrm{C}$. The proteins from the supernatant were measured using a bicinchoninic acid protein assay kit (Beyotime Institute of Biotechnology, Beijing, China). The proteins (20 $\mu \mathrm{g}$ per lane) were separated by $12 \%$ SDS-PAGE and transferred to a PVDF membrane (Merck KGaA, Darmstadt, Germany). The membrane was 
A

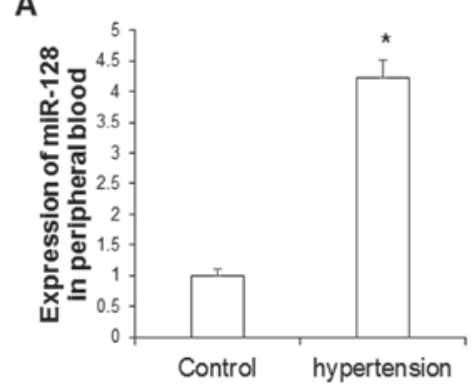

C
B

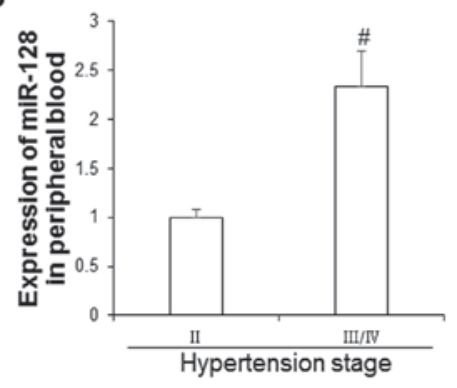

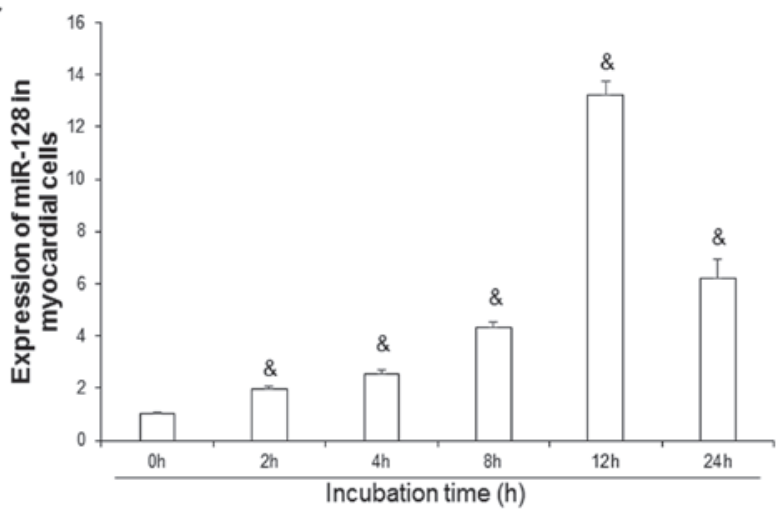

Figure 1. Expression of miR-128 in peripheral blood and injured myocardial cells as determined by RT-qPCR. (A) miR-128 expression was increased in hypertension peripheral blood compared with control blood. ${ }^{*} \mathrm{P}<0.05$, compared with hypertension peripheral blood. (B) miR-128 was increased in III/IV hypertension peripheral blood compared with II hypertension peripheral blood ${ }^{*} \mathrm{P}<0.05$, compared with III/IV hypertension peripheral blood. (C) miR-128 expression significantly increased with the extension of culture time in deprivation of oxygen and glucose. ${ }^{\&} \mathrm{P}<0.05$, compared with 0 h culture. miR, microRNA; $\mathrm{NC}$, negative controls.

blocked with $5 \%$ skim milk in TBST at room temperature for $1 \mathrm{~h}$. Membranes were subsequently incubated with the following primary antibodies at room temperature for $1 \mathrm{~h}$ : Rabbit anti-mouse c-Met polyclonal antibody (ab14570; 1:1,000; Abcam, Cambridge, UK) and anti-GAPDH (ab8245; 1:10,000; Abcam). Membranes were incubated with secondary antibodies for $1 \mathrm{~h}$ at room temperature, and subsequently washed 3 times with PBST. The secondary antibodies were horseradish peroxidase-conjugated goat anti-rabbit and goat anti-mouse immunoglobulin G (ab6721 and ab678, respectively; 1:5,000; Abcam). All antibodies were purchased from Santa Cruz Biotechnology, Inc. (Dallas, TX, USA). Finally, the membrane was developed using enhanced electrochemiluminescence Beyo ECL plus reagent (Beyotime Institute of Biotechnology).

Apoptosis detection in myocardial cells with different transfections. To elucidate the roles of miR-128 in myocardial injury, the apoptosis of cells in different groups was assessed; in cells transfected with miR-128 mimics, those transfected with c-Met siRNA and NCs. Apoptosis was detected using a FITC Annexin $\mathrm{V}$ Apoptosis Detection kit I (BD Biosciences) following the manufacturer's protocol and analyzed using BD FACSVerse flow cytometry (BD Biosciences). The FACS Diva software (version 4.0; BD Biosciences) was used to perform the analysis. Cells with positive Annexin V were determined to be in the early stage of apoptosis, cells with positive propdium iodide (PI) staining were undergoing necrosis, while cells with the positive Annexin V combined with positive PI were in the late stage of apoptosis.
Dual luciferase assay. Based on the bioinformatics prediction, the wild-type 3' untranslated region (UTR) and the mutant 3'UTR of c-Met were synthesized in vitro and were cloned into the downstream of pMIR-REPORT luciferase vector using the Spe-1 and HindIII enzymes (Beyotime Institute of Biotechnology). Human embryonic kidney (HEK) 293T cells (Shanghai Cell Bank; Chinese Academy of Sciences, Shanghai, China) were co-transfected with miR-128 mimics and wild-type c-Met 3'UTR or mutant 3'UTR. Following transfection for $24 \mathrm{~h}$, cells were lysed and luciferase intensity was measured using a GloMax 20/20 luminometer (Promega Corporation, Madison, WI, USA) based on the standard protocol of the Dual Luciferase Reporter Gene Assay kit (Beyotime Biotechnology). The intensity of Renilla was used as control and the fluorescence intensity in different groups was analyzed.

Statistical analysis. SPSS 19.0 software (IBM SPSS, Inc., Armonk, NY, USA) was used to perform statistical analysis. All data are presented as the mean \pm standard deviation and differences were determined by the two-tailed Student's t test. $\mathrm{P}<0.05$ was considered to indicate a statistically significant difference. All experiments were performed in triplicate.

\section{Results}

Expression of miR-128 in peripheral blood and injured myocardial cells. To examine miR-128 expression in peripheral blood, RT-qPCR was conducted. It was determined that miR-128 expression was significantly higher in patients with hypertension $(4.22 \pm 0.29)$ than controls $(\mathrm{P}<0.05$; Fig. 1A). 

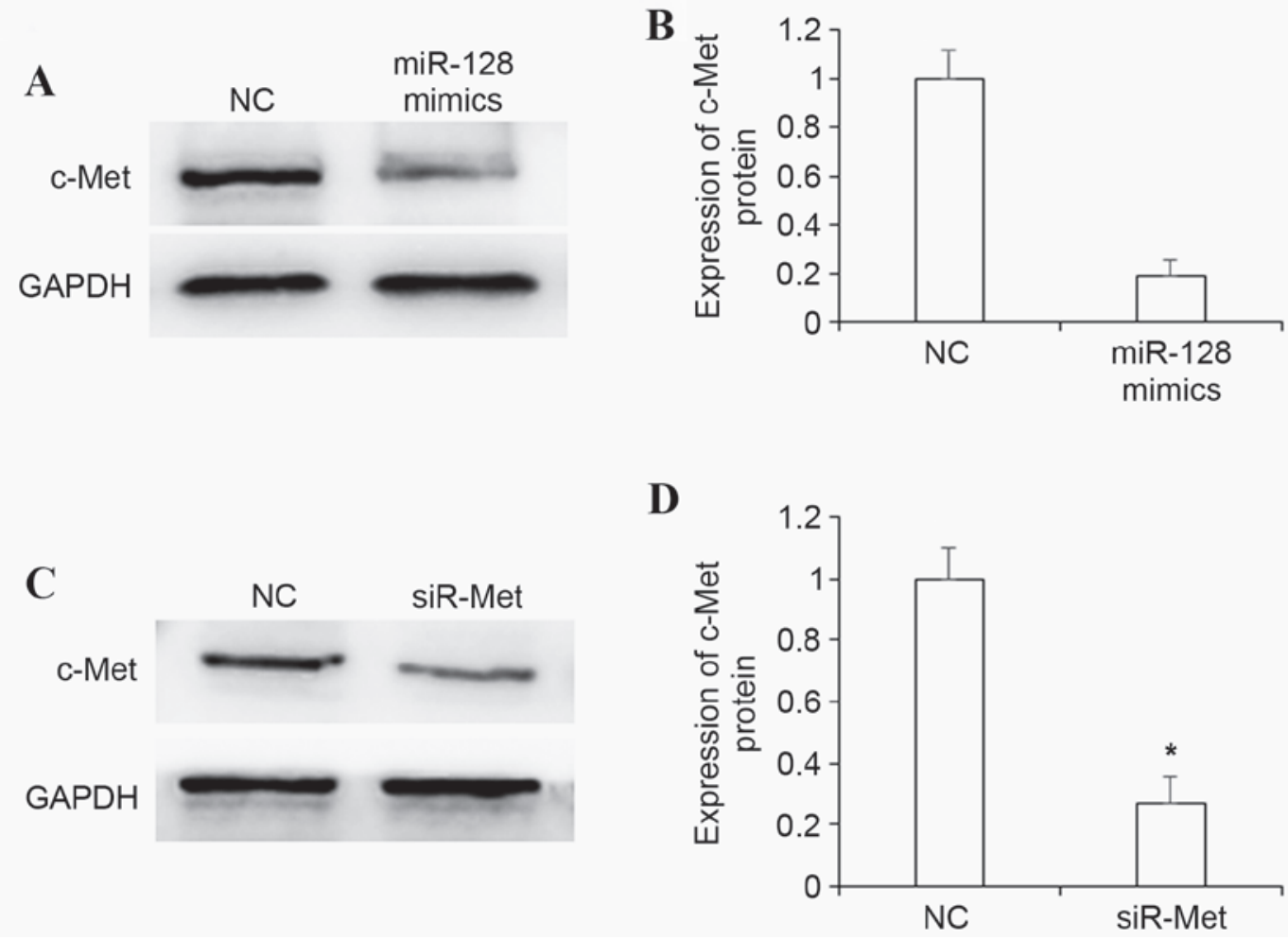

Figure 2. miR-128 repressed transcription of c-Met in myocardial cells. (A) Western blot measuring the expression of c-Met in myocardial cells transiently transfected with miR-128 mimic. (B) The expression of c-Met protein in cells transfected with miR-128 and NCs. "P $<0.05$, compared with NC group. (C) Western blot measuring the expression of c-Met protein in myocardial cells transiently transfected with siR-Met. (D) The expression of c-Met protein in cells transfected with siR-Met and NCs. "P<0.05 compared with NC group. miR, microRNA; siR, small interfering RNA; NC, negative controls.

miR-128 expression was also significantly upregulated in the peripheral blood of hypertension patients in III/IV stage (2.34 \pm 0.35$)$ compared with stage II patients (Fig. 1B).

To assess miR-128 expression in injured myocardial cells, myocardial cells were cultured under deprivation of oxygen or glucose. Cells were collected at $0,2,4,8,12$, and $24 \mathrm{~h}$ to detect the expression of miR-128. As shown in Fig. 1C, miR-128 expression significantly increased at $2 \mathrm{~h}$ compared with at $0 \mathrm{~h}(\mathrm{P}<0.05)$.

c-Met protein expression in myocardial cells as detected by western blot analysis. To detect the expression of c-Met protein, total proteins were extracted from myocardial cells transfected with either miR-128 mimics or c-Met siRNA. Expression of c-Met was significantly downregulated compared with NCs, when miR-128 was upregulated by miR-128 mimics $(\mathrm{P}<0.05$; Fig. 2A and B). Similarly, c-Met protein expression was significantly downregulated $(\mathrm{P}<0.05$; Fig. $2 \mathrm{C}$ and $\mathrm{D})$ when transfected with siRNA c-Met, compared with NCs.

c-Met is directly targeted by miR-128. To determine whether c-Met was directly regulated directly by miR-128, luciferase intensity was detected using a dual luciferase assay. The miR-128 seed sequence, the binding sites on c-Met 3'UTR and several base mutation were generated (Fig. 3A). It was determined that luciferase intensity significantly decreased following co-transfection with miR-128 mimics and wild-type c-Met 3'UTR, compared with NC $(\mathrm{P}<0.05)$. However, there was no significant difference between NC and mutant c-Met 3'UTR (P>0.05; Fig. 3B). These results indicate that miR-128 directly binds to the 'seed region' in the 3'UTR of c-Met mRNA.

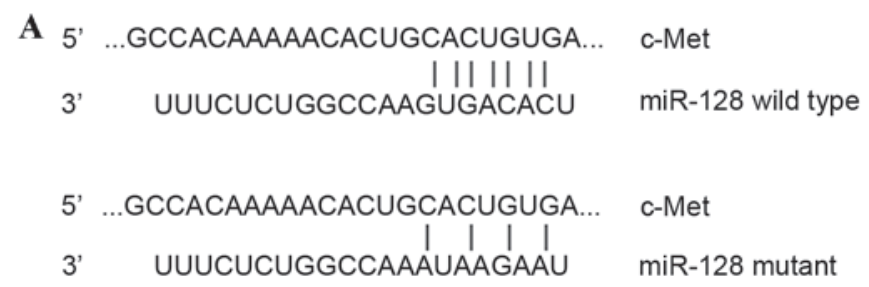

B

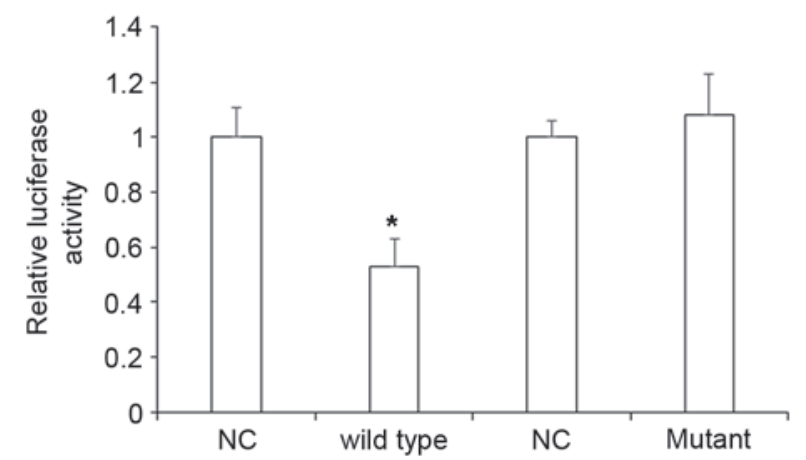

Figure 3. C-Met is a direct target gene of miR-128. (A) The wild type and mutant binding sites of miR-128 on c-Met 3'UTR. (B) Dual luciferase assay was used to detect whether $\mathrm{c}-\mathrm{Met}$ is regulated by miR-128. ${ }^{*} \mathrm{P}<0.05$, compared with NC. UTR, untranslated region; NC, negative control; miR, microRNA.

Apoptosis of myocardial cells regulated by miR-128 and c-Met. To detect cell apoptosis regulated by miR-128 and c-Met, flow cytometry was performed (Fig. 4). It was determined that the percentage of apoptotic cells was significantly higher 
A

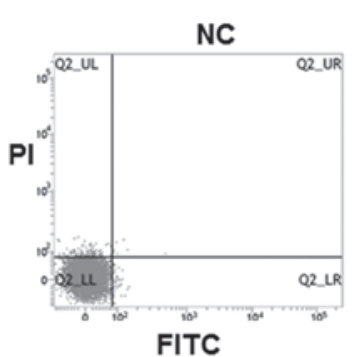

C

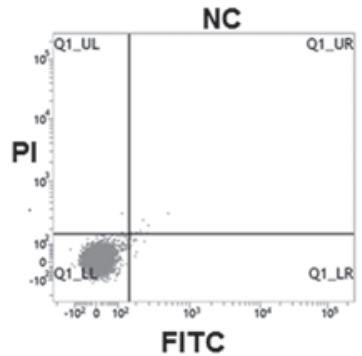

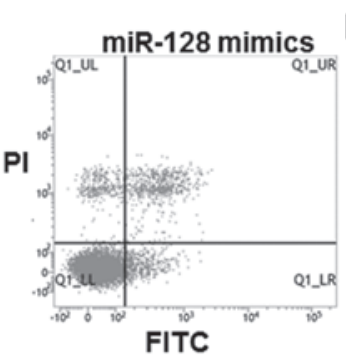

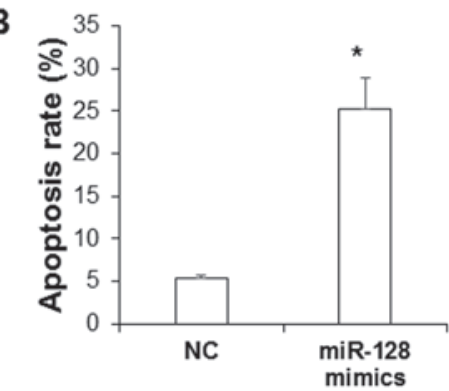

D
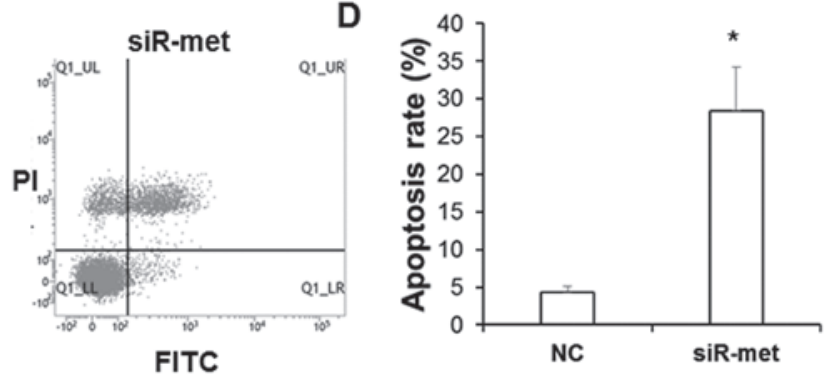

Figure 4. Apoptosis of myocardial cells regulated by miR-128 and c-Met. (A) Flow cytometry was used to detect the apoptosis of myocardial cells following transfection with miR-128 mimics. (B) Apoptosis rate of myocardial cells following transfection by miR-128 mimic compared with NCs. ${ }^{*}<0.05$ compared with NC. (C) Flow cytometry was used to detect the apoptosis of myocardial cells following transfection with siR-Met. (D) Apoptosis rate of myocardial cells following transfection with siR-Met compared with NCs. ${ }^{*} \mathrm{P}<0.05$, compared with NC. PI, propidium iodide; NC, negative control; SiR, small interfering RNA; miR, micro RNA; FITC, Fluorescein isothiocyanate.

in the miR-128 mimic group than the NC group $(\mathrm{P}<0.05$; Fig. 4B), indicating that miR-128 may induce the apoptosis of myocardial cells. When the expression of c-Met protein was downregulated by c-Met siRNA, the ratio of apoptosis was significantly higher $(\mathrm{P}<0.05$; Fig. 4D), indicating that $\mathrm{c}-\mathrm{Met}$ may protect myocardial cells. Taken together, these results suggest that miR-128 may promote the apoptosis of myocardial cells by increasing c-Met expression.

\section{Discussion}

Hypertension is an important risk factor for cardiovascular disease, as it influences cardiovascular structure and function (21). Furthermore, hypertension is the primary cause of mortality from cardiovascular disease, as it can induce heart failure (22). The development of molecular technology has allowed the mechanism of action of hypertension to be investigated at the molecular level (23) and recently, miRNA has been found to participate in the myocardial fibrosis, ventricular remodeling and myocardial injury induced by hypertension (10). It has been determined that the expression of many miRNAs in serum, including miR-1, miR-208 and miR-133, markedly change following acute myocardial infarction, suggesting that miRNA serves an important role in myocardial injury (24). By analyzing the expression of miRNA in the myocardial tissue of patients with heart failure using Next Generation Sequencing technology, Barsanti et al (25) found that miR-338-3p, miR-142 and 10 other types of small RNA were closely related to heart failure. Zhang et al (26) found that miR-206 was involved in the regulation of heart neural rhythm by targeting superoxide dismutase 1. Furthermore, Chen et al (27) identified that miR-214 expression promoted myocardial cell injury by downregulating itchy E3 ubiquitin protein ligase.
Previous studies have demonstrated that the HGF/c-Met signaling pathway serves key roles in myocardial fibrosis, ventricular remodeling and apoptosis $(28,29)$. Guo et al $(30)$ found that HGF expression was upregulated in cardiac tissue of rats that had experienced acute myocardial infarction. By inhibiting apoptosis and promoting angiogenesis, HGF can improve cardiac remodeling and heart function (30). Komamura et al (31) found that HGF overexpression significantly improved symptoms of hypertension and heart failure in a rat hypertension model, indicating that the HGF/c-Met signaling pathway may protect the myocardium.

The current study analyzed miR-128 expression in the peripheral blood of patients that had experienced hypertension for $>5$ years. It was demonstrated that miR-128 was upregulated in patients with hypertension compared with controls and that miR-128 expression was higher in patients with stage III/IV hypertension than in patients with stage II hypertension, indicating that miR-128 may be related with the progression of hypertension. Bioinformatic predictions showed that c-Met was one of the target genes of miR-128. Therefore, it may be hypothesized that miRNA-128 participates in myocardial cell injury by directly targeting c-Met, thus regulating the activity of the HGF/c-Met signaling pathway. To test this hypothesis, primary cardiomyocytes from neonatal rats were cultured in vitro under deprivation of oxygen and glucose to induce myocardial cell injury. Then, miR-128 expression was detected at different points of time. It was determined that miR-128 expression increased with the culture time and reached a peak at $12 \mathrm{~h}$. All these results indicate that miR-128 overexpression is related to the progress of myocardial injury.

Western blot analysis showed that the expression of c-Met protein decreased significantly and the ratio of apoptotic cells increased significantly following the transfection of miR-128 
mimics. The number of apoptotic cells also increased when c-Met expression was knocked down by siRNA. Based on these results, it may be hypothesized that miR-128 promotes myocardial cell injury by regulating the expression of c-Met. The results from the dual luciferase assay showed that the fluorescent intensity decreased significantly in the miR-128 mimics group, whereas there were no significant changes in the 3'UTR mutant group.

In conclusion, the current study has demonstrated that c-Met can be regulated by miR-128 through direct complementary binding to 3'-UTR. Therefore, miR-128 may inhibit the translation of c-Met mRNA and decrease the activity of HGF/c-Met signaling pathway to promote myocardial cell injury. miR-128 and c-Met, along with their associated signaling pathways, may be potential drug targets and therefore be of great clinical value.

\section{Acknowledgements}

The authors wish to thank Professor Jun Yang (Yuhangding Hospital of Yantai) for his guidance and assistance during research subject selection, experimental design, experiments, results analysis, and paper preparation and modification.

\section{References}

1. Szauder I, Csajági E, Major Z, Pavlik G and Ujhelyi G: Treatment of hypertension: Favourable effect of the twice-daily compared to the once-daily (Evening) administration of perindopril and losartan. Kidney Blood Press Res 40: 374-385, 2015.

2. Nagiub M, Kanaan U, Simon D and Guglani L: Risk factors for development of pulmonary hypertension in infants with bronchopulmonary dysplasia: Systematic review and meta-analysis. Paediatr Respir Rev: Nov 22, 2016 (Epub ahead of print). doi 10.1016/j.prrv.2016.11.003.

3. Poulter NR, Prabhakaran D and Caulfield M: Hypertension. Lancet 386: 801-812, 2015.

4. Cloutier L, Daskalopoulou SS, Padwal RS, Lamarre-Cliche M, Bolli P, McLean D, Milot A, Tobe SW, Tremblay G, McKay DW, et al: A new algorithm for the diagnosis of hypertension in Canada. Can J Cardiol 31: 620-630, 2015.

5. Ma WF, Liang Y, Zhu J, Yang YM, Tan HQ, Yu LT, Gao X, Feng GX and Li JD: Comparison of 4 admission blood pressure indexes for predicting 30-day mortality in patients with ST-segment elevation myocardial infarction. Am J Hypertens 29: 332-339, 2016

6. Rasmussen JT, Thenappan T, Benditt DG, Weir EK and Pritzker MR: Is cardiac resynchronization therapy for right ventricular failure in pulmonary arterial hypertension of benefit? Pulm Circ 4: 552-559, 2014.

7. Kannan A and Janardhanan R: Hypertension as a risk factor for heart failure. Curr Hypertens Rep 16: 447, 2014.

8. Charkoudian $\mathrm{N}$ and Wallin BG: Sympathetic neural activity to the cardiovascular system: Integrator of systemic physiology and interindividual characteristics. Compr Physiol 4: 825-850, 2014.

9. Zhang $\mathbf{J}$ and Crowley SD: Role of T lymphocytes in hypertension. Curr Opin Pharmacol 21: 14-19, 2015.

10. Romaine SP, Tomaszewski M, Condorelli G and Samani NJ: MicroRNAs in cardiovascular disease: An introduction for clinicians. Heart 101: 921-928, 2015.

11. Neves VJ, Fernandes T, Roque FR, Soci UP, Melo SF and de Oliveira EM: Exercise training in hypertension: Role of microRNAs. World J Cardiol 6: 713-727, 2014

12. van den Hoogen P, van den Akker F, Deddens JC and Sluijter JP: Heart failure in chronic myocarditis: A role for microRNAs? Curr Genomics 16: 88-94, 2015.
13. Zhou J, Gao J, Zhang X, Liu Y, Gu S, Zhang X, An X, Yan J, Xin Y and Su P: microRNA-340-5p functions downstream of cardiotrophin-1 to regulate cardiac eccentric hypertrophy and heart failure via target gene dystrophin. Int Heart J 56: 454-458, 2015.

14. Witman N, Heigwer J, Thaler B, Lui WO and Morrison JI: miR-128 regulates non-myocyte hyperplasia, deposition of extracellular matrix and Islet1 expression during newt cardiac regeneration. Dev Biol 383: 253-263, 2013.

15. Gao F, Deng G, Liu W, Zhou K and Li M: Resveratrol suppresses human hepatocellular carcinoma via targeting HGF-c-Met signaling pathway. Oncol Rep 37: 1203-1211, 2017.

16. Sala V and Crepaldi T: Novel therapy for myocardial infarction: Can HGF/Met be beneficial? Cell Mol Life Sci 68: 1703-1717, 2011.

17. Li H, Zhang H, Zhao S, Shi Y, Yao J, Zhang Y, Guo H and Liu X: Overexpression of MACC1 and the association with hepatocyte growth factor/c-Met in epithelial ovarian cancer. Oncol Lett 9: 1989-1996, 2015

18. Goyal L, Zheng H, Yurgelun MB, Abrams TA, Allen JN, Cleary JM,Knowles M, ReganE, Reardon A, Khachatryan A, etal: A phase 2 and biomarker study of cabozantinib in patients with advanced cholangiocarcinoma. Cancer: Feb 13, 2017 (Epub ahead of print).

19. Monteiro MB, Santos-Bezerra DP, Thieme K, Passarelli M, Machado UF, Lin CJ and Corrêa-Giannella ML: Optimization of total RNA isolation from human urinary sediment. Clin Chim Acta 462: 158-161, 2016.

20. Livak KJ and Schmittgen TD: Analysis of relative gene expression data using real-time quantitative PCR and the 2(-Delta Delta C(T)) Method. Methods 25: 402-408, 2001.

21. Del Gobbo LC, Kalantarian S, Imamura F, Lemaitre R, Siscovick DS, Psaty BM and Mozaffarian D: Contribution of major lifestyle risk factors for incident heart failure in older adults: The cardiovascular health study. JACC Heart Fail 3: 520-528, 2015.

22. Rosenkranz S, Ghofrani HA, Beghetti M, Ivy D, Frey R, Fritsch A, Weimann G, Saleh S and Apitz C: Riociguat for pulmonary arterial hypertension associated with congenital heart disease. Heart 101: 1792-1799, 2015.

23. Watson CJ, Horgan S, Neary R, Glezeva N, Tea I, Corrigan N, McDonald K, Ledwidge M and Baugh J: Epigenetic therapy for the treatment of hypertension-induced cardiac hypertrophy and fibrosis. J Cardiovasc Pharmacol Ther 21: 127-137, 2016.

24. Sayed AS, Xia K, Yang TL and Peng J: Circulating microRNAs: A potential role in diagnosis and prognosis of acute myocardial infarction. Dis Markers 35: 561-566, 2013.

25. Barsanti C, Trivella MG, D'Aurizio R, El Baroudi M, Baumgart M, Groth M, Caruso R, Verde A, Botta L, Cozzi L and Pitto L: Differential regulation of microRNAs in end-stage failing hearts is associated with left ventricular assist device unloading. Biomed Res Int 2015: 592512, 2015.

26. Zhang Y, Zheng S, Geng Y, Xue J, Wang Z, Xie X, Wang J, Zhang S and Hou Y: MicroRNA profiling of atrial fibrillation in canines: MiR-206 modulates intrinsic cardiac autonomic nerve remodeling by regulating SOD1. PLoS One 10: e0122674, 2015.

27. Chen ZG, Liu H, Zhang JB, Zhang SL, Zhao LH and Liang WQ: Upregulated microRNA-214 enhances cardiac injury by targeting ITCH during coxsackievirus infection. Mol Med Rep 12: 1258-1264, 2015.

28. Akiyama Y, Ashizawa N, Seto S, Ohtsuru A, Kuroda H, Ito M, Yamashita S and Yano K: Involvement of receptor-type tyrosine kinase gene families in cardiac hypertrophy. J Hypertens 17: 1329-1337, 1999.

29. Ono K, Matsumori A, Shioi T, Furukawa Y and Sasayama S: Enhanced expression of hepatocyte growth factor/c-Met by myocardial ischemia and reperfusion in a rat model. Circulation 95: 2552-2558, 1997.

30. Guo Y, He J, Wu J, Yang L, Dai S, Tan X and Liang L: Locally overexpressing hepatocyte growth factor prevents post-ischemic heart failure by inhibition of apoptosis via calcineurin-mediated pathway and angiogenesis. Arch Med Res 39: 179-188, 2008.

31. Komamura K, Miyazaki J, Imai E, Matsumoto K, Nakamura T and Hori M: Hepatocyte growth factor gene therapy for hypertension. Methods Mol Biol 423: 393-404, 2008. 